\title{
Narrative review of portal vein thrombosis in cirrhosis: pathophysiology, diagnosis, and management from an interventional radiology perspective
}

\author{
Sameer Gadani ${ }^{1 \wedge}$, Sasan Partovi ${ }^{1 \wedge}$, Abraham Levitin ${ }^{1 \wedge}$, Nicholas Zerona $^{1} \wedge$, Shreya Sengupta $^{2}$, \\ Giuseppe D’Amico ${ }^{2} \wedge$, Teresa Diago Uso ${ }^{2}$, K. V. Narayanan Menon ${ }^{2}$, Cristiano Quintini ${ }^{2} \wedge$ \\ ${ }^{1}$ Imaging Institute, Cleveland Clinic Foundation, Cleveland, OH, USA; ${ }^{2}$ Digestive Disease and Surgery Institute, Cleveland Clinic Foundation, \\ Cleveland, OH, USA \\ Contributions: (I) Conception and design: S Gadani, S Partovi, N Zerona; (II) Administrative support: S Gadani, S Partovi, N Zerona; (III) Provision \\ of study materials or patients: S Gadani, S Partovi, N Zerona; (IV) Collection and assembly of data: S Gadani, S Partovi, N Zerona; (V) Data analysis \\ and interpretation: S Gadani, S Partovi, N Zerona; (VI) Manuscript writing: All authors; (VII) Final approval of manuscript: All authors. \\ Correspondence to: Sameer Gadani, MD. Cleveland Clinic Imaging Institute, 9500 Euclid Ave., Cleveland, Ohio 44195, USA.
}

Email: GADANIS@ccf.org.

Objective: This paper examines the incidence, clinical presentation, and pathophysiology of portal vein thrombosis (PVT) in cirrhosis. Additionally, we have reviewed the literature regarding the current status of medical and interventional radiology management of PVT and have proposed a novel algorithm for the management given different clinical scenarios. Lastly two representative cases displaying endovascular treatment options are provided.

Background: Portal vein thrombus in the setting of cirrhosis is an increasingly recognized clinical issue with debate on its pathophysiology, natural course, and optimal treatment. Approximately one-third of patients are asymptomatic, and detection of the thrombus is an incidental finding on imaging performed for other reasons. In $30 \%$ to $50 \%$ of patients, PVT resolves spontaneously. However, there is increased posttransplant mortality in patients with completely occlusive PVT, therefore effective early revascularization strategies are needed for patients with complete PVT who are expected to undergo liver transplant. Additionally, no consensus has been reached regarding PVT treatment in terms of timing and type of interventions as well as type and duration of anticoagulation.

Methods: Computerized literature search as well as discussion with experts in the field.

Conclusions: Management of PVT is complex, as many variables affect which treatments can be used. Anticoagulation appears to be the optimal first-line treatment in patients with acute PVT but without bleeding varices or mesenteric ischemia. Minimally invasive treatments include various methods of mechanical thrombectomy, chemical thrombolysis, and transjugular intrahepatic portosystemic shunt (TIPS) placement with or without variceal embolization. Definitive recommendations are difficult due to lack of high quality data and continued research is needed to evaluate the efficacy of different anticoagulants as well as the timing and use of various minimally invasive therapies in specific circumstances.

Keywords: Cirrhosis; intervention; portal vein thrombosis (PVT); narrative review

Submitted Feb 24, 2021. Accepted for publication Oct 25, 2021.

doi: $10.21037 / \mathrm{cdt}-21-98$

View this article at: https://dx.doi.org/10.21037/cdt-21-98

\footnotetext{
^ ORCID: Sameer Gadani, 0000-0001-9200-8410; Sasan Partovi, 0000-0002-0856-6928; Abraham Levitin, 0000-0002-0089-8231; Nicholas Zerona, 0000-0002-9611-1404; Giuseppe D’Amico, 0000-0002-6004-9311; Cristiano Quintini, 0000-0002-6802-1436.
} 


\section{Introduction}

Portal venous thrombosis (PVT) is defined as a thrombus in the lumen of the portal vein. PVT is an increasingly recognized problem in the setting of cirrhosis. It can range from partial and asymptomatic obstruction of the vein to complete obstruction leading to hepatic decompensation, variceal bleeding, and intestinal infarction. In addition to cirrhosis leading to portal flow stasis, inherited or acquired prothrombotic diseases and vascular endothelial injury due to abdominal infection, surgery, or trauma can lead to portal and mesenteric venous thrombosis (1). In patients with PVT and cirrhosis, it is unclear whether the presence of PVT is caused by worsening liver cirrhosis or whether the thrombus itself is causing worsening of liver function. Based on available data, PVT appears to occur more frequently as liver function worsens (2-4). This suggests that PVT could be a marker of disease severity rather than a causative factor for further decompensation of liver function.

Although PVT is a known phenomenon in patients with cirrhosis, we have limited understanding of its pathophysiology and natural course and of how to prevent and treat this condition. In approximately $30 \%$ to $50 \%$ of patients, PVT resolves spontaneously. However, research has demonstrated increased post-transplant mortality in patients with completely occlusive PVT (4). Thus, effective early revascularization strategies are needed for patients with complete PVT who are expected to undergo liver transplant (5). For other patients, however, no consensus has been reached regarding PVT treatment in terms of timing and type of interventions as well as type and duration of anticoagulation. In this article, we will discuss the current status of medical and interventional radiology management of PVT.

A computerized literature search for was conducted in MEDLINE and PubMed for relevant English language articles in conjunction with discussions with experts in the field. We present the following article in accordance with the Narrative Review reporting checklist (available at https:// cdt.amegroups.com/article/view/10.21037/cdt-21-98/rc).

\section{Incidence, clinical presentation, and classification}

The incidence of PVT in the setting of cirrhosis is reported to range from $0.6 \%$ to $26 \%$, with increased rates seen in patients with decompensated cirrhosis (6,7). A multicenter study in 753 patients with cirrhosis reported a prevalence of ultrasound-documented PVT of $17 \%$, with an annual incidence rate of 6.05 and an increased incidence of PVT in patients with a similar prior history (1).

The clinical presentation of PVT is nonspecific. Approximately one-third of patients are asymptomatic and detection of the thrombus is an incidental finding on imaging performed for other reasons. In symptomatic patients, the symptoms are dependent on location, severity, and age of the thrombus. Acute PVT can present with an acute abdomen, non-bloody diarrhea, or ileus when associated with extension in the superior mesenteric vein (SMV). Frequently, PVT presents with upper gastrointestinal bleed due to portal gastropathy specifically when there is pre-existing portal hypertension. Acute SMV or inferior mesenteric vein thrombosis may present with acute abdomen with mesenteric ischemia. In such patients, 30-day mortality is $20 \%$. Overall, PVT accounts for $6 \%$ to $15 \%$ of cases of acute mesenteric ischemia (8-10). Chronic PVT usually presents with signs of portal hypertension including upper gastrointestinal bleeding secondary to esophageal and gastric varices and/or portal gastropathy, splenomegaly with pancytopenia, and ascites. Rarely, patients may present with cholestasis from portal bilopathy due to cavernous transformation of the portal vein $(11,12)$.

There are many different classification systems to standardize the description of PVT. Some of them address only the anatomic location and severity of the thrombus while others take into account the functional and clinical status of the patient. One of the more frequently used classification systems is the Yerdel classification system (13), which is used to make management decisions during surgical procedures including liver transplantation. However, this system is only an anatomic classification system and does not address the age of thrombus or provide functional information.

Considering the limitations of the Yerdel classification and other similar anatomic classifications, a new classification system that includes functional information has been proposed. In addition to the anatomical location, this anatomico-functional classification takes into account the degree of occlusion, duration and presentation, extent of thrombus, and presence and type of underlying liver disease (14).

\section{Pathophysiology and etiology}

Virchow's triad of hypercoagulability, stasis of blood flow, and endothelial injury leading to thrombosis is present in the portal and mesenteric venous system in the setting of 
cirrhosis, specifically in decompensated cirrhosis (12).

Patients with cirrhosis have a hypercoagulable state evidenced by normal or increased thrombin levels due to altered hemostatic balance. Patients have decreased levels of anticoagulation factors such as antithrombin and protein $\mathrm{C}$ with increased concentrations of procoagulation factors such as factor VIII and von Willebrand factor (vWF). Although thrombocytopenia is typical, the thrombogenic potential of platelets is increased due to decreased $\mathrm{vWF}$ cleaving protease ADAMTS-13, leading to altered interaction of vWF with platelets $(1,15,16)$. Endotoxemia secondary to bacterial translocation from the intestinal mucosa to the portal vein, which is frequently seen in patients with cirrhosis, also leads to a prothrombotic state $(1,2,17)$.

Parenchymal architectural distortion leads to increased vascular resistance with decreased flow. Additionally, portosystemic collateral circulation and splanchnic vasodilatation contribute to further stagnation of the flow. This decrease in portal venous flow is associated with PVT. Blood flow of less than $15 \mathrm{~cm} / \mathrm{s}$ in the portal vein is associated with increased risk of PVT $(6,18)$. Endothelial injury in cirrhotic patients could be due to spontaneous bacterial peritonitis, systemic infections, hepatic resection, endoscopic variceal sclerotherapy, transjugular intrahepatic portosystemic shunt (TIPS) placement, or other endovascular portal venous interventions. These can contribute to PVT as well. In addition, hepatocellular carcinoma (HCC) or macrovascular invasion could lead to PVT $(2,19)$. Nonalcoholic steatohepatitis (NASH) cirrhosis appears to be a potential risk factor for PVT, since it is associated with increased plasminogen activator inhibitor and reduced protein $\mathrm{C}$ levels $(1,2)$.

\section{Diagnosis}

The role of serum markers for PVT prediction in patients with cirrhosis is uncertain. Levels of D-dimers and protein $\mathrm{C}$ and $\mathrm{S}$ are proposed as potential predictors of PVT, although considering the significant heterogeneity amongst studies, generalizable conclusions cannot be made (20,21). Sarin et al. proposed a pretest probability assessment for PVT based on 10 criteria, including clinical, laboratory, and imaging components. Three major criteria include Child-Pugh B or C class, history of PVT, and prothrombotic risk factors (e.g., factor V Leiden mutation, prothrombin gene mutation, MTHFR mutation). Seven minor criteria include large portosystemic shunt, gastric varices, HCC, previous or active systemic venous thrombotic events or abortions, acute abdomen, new onset or worsening of portal hypertension complications, recent endoscopic/surgical/radiologic interventions, and portal flow velocity less than $15 \mathrm{~cm} / \mathrm{s}$. This proposed 10 -point assessment for the prediction of PVT appears to be comprehensive, but not has been validated in prospective trials (14).

Frequently, PVT is an incidental finding in patients with cirrhosis who are undergoing routine imaging surveillance for HCC. Ultrasound with Doppler evaluation of the liver is a frequently used initial test to identify the presence of PVT. Ultrasound is low cost, widely available, and does not expose the patient to radiation. It is $89 \%$ sensitive and $92 \%$ specific when compared with gold-standard angiographic or surgical-pathologic findings. Limitations of ultrasound include the patient's body habitus and intestinal gas interfering with the examination, specifically when evaluating PVT extension into the mesenteric and splenic veins. Additionally, ultrasound is operator dependent, requiring an experienced sonographer. Crosssectional imaging techniques such as CT angiography and MR angiography are more sensitive and specific then Doppler imaging (90\% sensitive and 99\% specific versus the gold standard). They provide information regarding age, size, and extent of the thrombus within the portal vein. Additionally, they identify extension of the thrombus in the superior mesenteric and/or splenic vein, provide information regarding the effect on extravascular structures such as bowel and mesentery, and delineate collaterals and portosystemic shunts $(8,22)$. CT angiography can be performed quickly; however, its use can be limited in patients with compromised renal function and allergy to iodinated contrast. MR angiography is performed when CT angiography is contraindicated. MR imaging provides better soft tissue resolution and does not involve ionizing radiation; additionally, the paramagnetic contrast agents used in MR imaging have a good safety profile. MR imaging is limited by longer acquisition times (30-60 min) and lower spatial resolution when compared with CT (23). Typically, ultrasound with Doppler is performed first in cases of suspected PVT. If the diagnosis is confirmed on ultrasound, cross-sectional imaging such as CT angiography or MR angiography (if CT angiography is contraindicated) is performed to characterize and map the extent of the thrombus, to determine its effect on the extravascular structures, and to identify portosystemic collaterals. 


\section{Management of acute PVT}

Optimal management of PVT in cirrhosis remains unclear without definite recommendations. Anticoagulation appears to be the initial therapy of choice, with the goals of preventing thrombus extension or recurrence, establishing vessel patency, and preventing complications of thrombosis such as portal hypertension and intestinal ischemia/ infarction. In the following sections, we will discuss various medical and interventional management options.

\section{Anticoagulation}

The decision to use anticoagulation for PVT in cirrhotic patients requires careful consideration of bleeding risk due to portal hypertension, severity of cirrhosis, and potential benefits of recanalization of thrombosed vessel. In a retrospective study by Noronha Ferreira et al. (5), bleeding in patients with portal hypertension receiving anticoagulation did not increase rebleeding rate, duration of hospitalization, 6-week mortality, or the need for rescue therapy such as TIPS placement. In a study by Loffredo et al. (24), the incidence of major and minor bleeding was the same in cirrhotic patients whether or not they received anticoagulation. In fact, the authors found a significantly lower incidence of variceal bleeding in patients on anticoagulation compared to those not on anticoagulation. Similar findings were also noted in multiple other retrospective studies $(25,26)$.

PVT recanalization rates are higher in patients who receive anticoagulation than in those who do not (51.4\% vs. $18.8 \%, \mathrm{P}=0.005$ ) (24). Similar recanalization rates with anticoagulation have been reported in the literature (ranging from $33-75 \%)(5,24,26)$. In a multivariate analysis, Noronha Ferreira et al. (5) found baseline Model For End-Stage Liver Disease (MELD) score to be an important predictor of recanalization rate, suggesting a beneficial effect of anticoagulation in patients with more advanced cirrhosis. Similar findings were reported in a study by Pettinari et al. (27). This study demonstrated that orthotopic liver transplant-free survival was significantly higher in ChildPugh class B patients, and a similar trend was noted in ChildPugh class $\mathrm{C}$ patients. This could be due to more pronounced portal hemodynamic changes and imbalances in procoagulant factors in patients with advanced cirrhosis, which could be caused by endothelial dysfunction and bacterial translocation. The beneficial effect of anticoagulation could be due to its effect on the hepatic microvascular thrombosis secondary to inflammation and/or fibrosis-fibrin deposition in patients with advanced cirrhosis $(28,29)$.

The re-thrombosis rate in patients who stopped anticoagulation was $35.3 \%$ in a study by Noronha Ferreira et al. (5), which correlates with findings from previous studies $(27,30)$. These data reinforce that a permanent prothrombotic state exists in the portal venous system of cirrhotic patients, which justifies maintaining anticoagulation indefinitely in the absence of contraindications (31).

PVT does not influence transplant waiting list mortality. However, the presence of PVT (specifically occlusive and extensive) at the time of orthotopic liver transplant is associated with longer operative times, higher blood transfusion requirements, and increased risk of mortality and the need for post-transplant interventions. Therefore, anticoagulation is recommended in patients with cirrhosis and PVT who are on the transplant waiting list (32).

With limited clinical data, there is a lack of consensus regarding the ideal anticoagulation regimen for PVT in cirrhotic patients (33). Inpatients are typically prescribed weight-based intravenous heparin due to its wide availability, short half-life, reversibility, and clinical efficacy (11). For outpatients, a variety of options are available, including vitamin $\mathrm{K}$ antagonists, low-molecular-weight heparin, direct/ newer anticoagulants, and warfarin. Vitamin $\mathrm{K}$ antagonists are orally administered and have the advantage of being reversible and inexpensive; additionally, the data supporting the use of these agents in cirrhotic patients are strong. However, they are limited by their effect on MELD score and the need for INR monitoring (34). Low-molecularweight heparin is administered as a fixed dose and does not require monitoring. This agent does not have an effect on MELD score and is easily reversible, again with strong data supporting its use in cirrhosis. However, it is limited by cost, the need for parenteral administration (potentially leading to poor compliance), and its effect on renal function. Newer anticoagulants are administered orally at a fixed dose, without the need for monitoring and with minimal effect on MELD score. Unfortunately, there are limited data regarding the use of these agents in cirrhosis; they are also expensive, and no approved reversal agents are currently available $(35,36)$. A detailed discussion regarding the various anticoagulation agents for PVT in cirrhosis is beyond the scope of this review.

\section{Endovascular treatments}

Many endovascular treatment options are available for acute PVT in cirrhosis; these treatments are used in isolation or 
in conjunction with systemic anticoagulation. These therapy options include mechanical thrombectomy and/or chemical thrombolysis with either percutaneous or transjugular transhepatic portal venous access, TIPS placement with endovascular management of esophageal and gastric varices, and recanalization of chronically occluded PVT with TIPS placement. Frequently, these endovascular treatments are used in conjunction with each other.

\section{Mechanical thrombectomy}

There are variety of mechanical thrombectomy devices available commercially. They are typically used in conjunction with catheter-directed thrombolysis, as it is theoretically advantageous to reduce the thrombus burden, resulting in reduced requirement for a thrombolytic in terms of volume and duration and thus reducing the risk of bleeding. The mechanical thrombectomy options available include hydrodynamic thrombectomy (maceration of the thrombus with aspiration), balloon thrombectomy, and thrombectomy via aspiration devices. For thrombectomy, the portal venous system can be accessed via transjugular, transhepatic or percutaneous transhepatic, or transplenic routes. Percutaneous transjugular transhepatic access ensures no organ capsular disruption with a relatively low internal bleeding risk. This access also allows relatively larger access sheath placement for a variety of thrombectomy devices. However, obtaining transjugular access to the thrombosed portal vein can be challenging. This can be accomplished by using intravascular or percutaneous ultrasound guidance, which can precisely guide needle access from the hepatic vein to the portal vein $(37,38)$.

One commonly used thrombectomy device is the Angiojet device. This is a pharmacomechanical thrombectomy device that macerates the thrombus and clears it through the rheolytic effect (Bernoulli principle). The Angiojet catheter is available in 6 and 8 French sizes. Considering the size of the portal vein, an 8 French Angiojet is the preferred catheter. The device generates a high-speed retrograde fluid jet at the tip that creates high shear gradient through the Venturi effect, which fragments the thrombus. Debris are immediately evacuated through the lumen of the catheter $(39,40)$. The thrombus can be laced with tPA before the thrombectomy is performed, or the Angiojet can be used in "power pulse mode" to distribute the thrombolytic agents within the thrombus (Figure 1). Similar but less frequently used hydrodynamic thrombectomy devices are the Hydrolyser and Oasis devices. These mechanical thrombectomy devices reduce the required dose and length of infusion time for subsequent thrombolysis, reducing the risk of bleeding and expediting the patient's recovery by rapidly reducing intestinal congestion $(40,41)$.

Aspiration thrombectomy has evolved from a basic large-volume high-pressure syringe and large-bore catheter to vacuum-assisted systems. The Indigo aspiration thrombectomy system (Penumbra) is a continuous suction device that is frequently used in neurovascular and peripheral applications. Of the various catheter sizes, 8 French is most commonly used in the portal venous system. With this system, a significant amount of blood loss can occur in a short time period if the catheter tip is not engaged with the thrombus. However, with the use of the automatic valve control in the new Lightning Aspiration Tubing of the catheter system, thrombus aspiration is optimized and the risk of blood loss is reduced.

A new mechanical and aspiration thrombectomy device called FlowTriever Mechanical Thrombectomy (Inari Medical) is now available. The aspiration catheters for this system are 16, 20 or 24 French and use large syringes attached to the side arm to create negative pressure within the catheter to aid in aspiration of the thrombus from large vessels. Additionally, newer FlowTriever catheters with discs of various sizes are also available to help capture and remove adherent thrombi. These catheter systems require a large access sheath (up to 24 French), which precludes their use in PVT if a TIPS is not available as access to the portal venous system (42) (Figure 2). A balloon angioplasty can also be used for thrombectomy, specifically when the thrombus burden is small. This method is often used in conjunction with other mechanical thrombectomy techniques.

\section{Catheter-directed thrombolysis}

Catheter-directed thrombolysis involves infusion of the thrombolytic medicine directly into the thrombus, frequently in conjunction with mechanical thrombectomy. This technique is routinely used in other anatomic locations, including for the treatment of deep vein thrombosis in peripheral veins, inferior vena cava thrombus, and acute pulmonary embolism (43). The portal vein can be accessed through either the transjugular or percutaneous transhepatic route. Either of these accesses can be used to place an infusion catheter in the thrombus. These infusion catheters (Cragg-McNamara, Medtronic, Minneapolis, MN; or UniFuse, Angiodynamics, Latham, NY, USA) are 4 or 5 

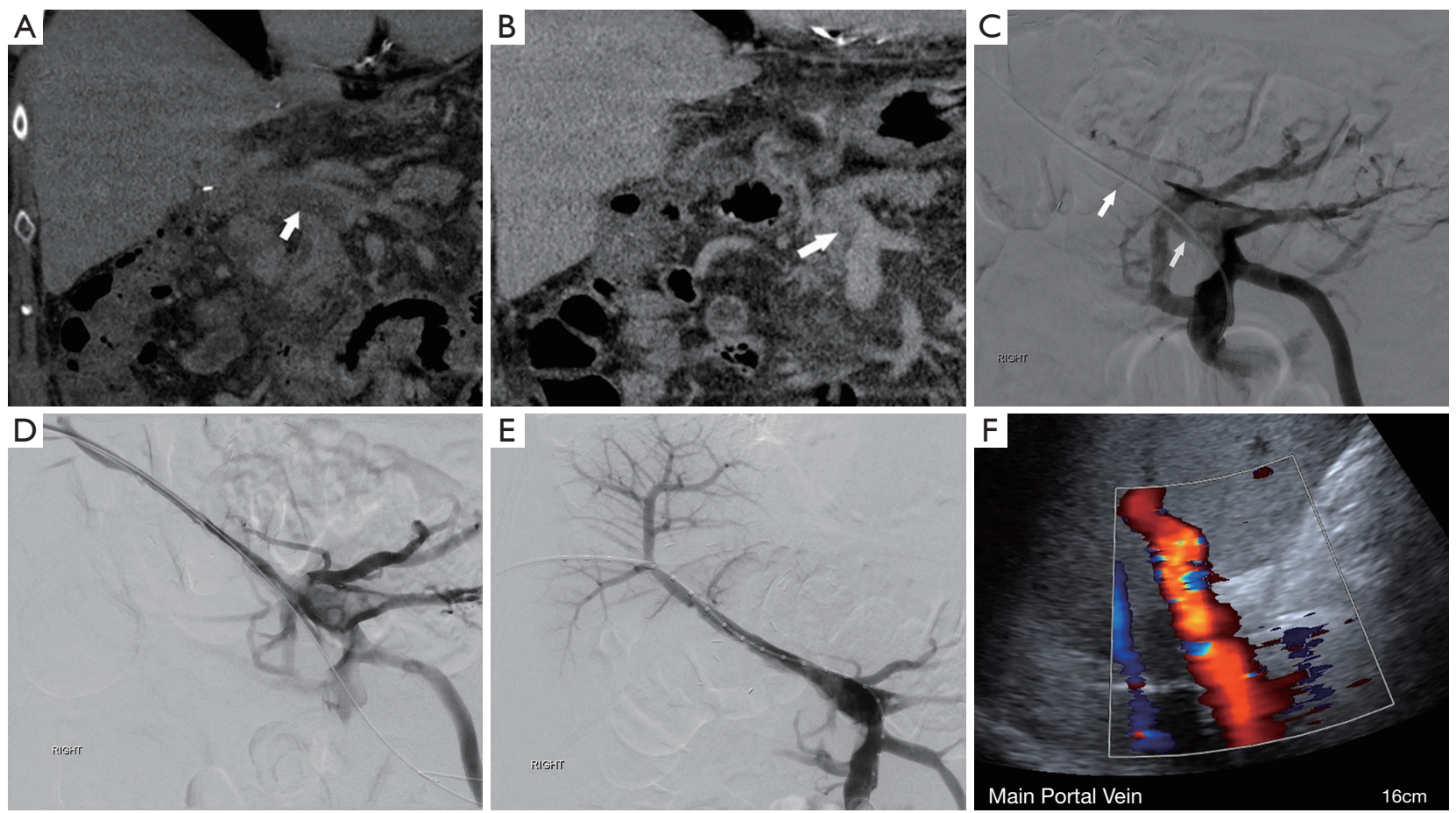

Figure 1 Case 1: M, 62, with NASH cirrhosis and HCC in the left lobe of the liver status post left lobe of the liver resection. Patient developed PVT. (A and B) demonstrating main portal vein thrombosis extending in to the SMV (both marked by arrow). (C) is a portal venogram through transhepatic access in frontal projection. It shows complete occlusion of the main portal vein with partially occlusive thrombus extending in to the confluence and SMV (marked by arrows). (D) was obtained after lacing the portal venous thrombus with alteplase and balloon venoplasty. It reveals diminutive recanalized portal vein with poor intrahepatic flow. (E) obtained after Angiojet (8 French catheter) mechanical thrombectomy. It shows marked improvement in the intra and extrahepatic portal vein flow. (F) is a color Doppler ultrasound obtained after six months confirming patency of the portal vein. NASH, nonalcoholic steatohepatitis; HCC, hepatocellular carcinoma; SMV, superior mesenteric vein; PVT, portal venous thrombosis.

French and have multiple side holes in the infusion length of the catheter with a tip occluding guidewire. This allows preferential flow of the fibrinolytic medication (alteplase, Genentech, South San Francisco, CA, USA) at a specific rate directly within the thrombus. The rate and length of infusion depend on the thrombus burden; however, alteplase is typically infused at a rate of $1 \mathrm{mg} / \mathrm{h}$ for up to 24 hours. During infusion of the fibrinolytic medication, patients are typically monitored in the intensive care unit for signs of bleeding. Laboratory surveillance of fibrinogen levels (should remain above $150 \mathrm{mg} / \mathrm{dL}$ ), coagulation parameters, and hemoglobin/hematocrit levels is also used to titrate the dose of fibrinolytic medication and to determine the length of infusion. Considering the potential for bleeding due to thrombolysis, transjugular transhepatic access is preferred over percutaneous access $(44,45)$. Additionally, ultrasoundassisted thrombolytic infusion (EKOS) has been proposed, with potentially accelerated thrombolysis to reduce the infusion time and the fibrinolytic dose. However, the potential benefits of this technique when compared with conventional thrombolysis have not been validated in the literature. After thrombectomy and thrombolysis, patients should undergo anticoagulation and imaging surveillance to assess for recurrence of PVT (46).

There are limited data in the literature regarding the role of thrombolysis in patients with PVT and cirrhosis. In a retrospective review of 17 patients with noncirrhotic, nonmalignant PVT who had undergone transjugular transhepatic thrombolysis with or without TIPS placement, 94.1\% technical success (defined as complete or partial recanalization) was reported (43). Cumulative percentages for primary and secondary patency of the portal vein at 24 months were $74 \%$ and $88.2 \%$, respectively. Clinical success was demonstrated by prevention of resection in 15 of 

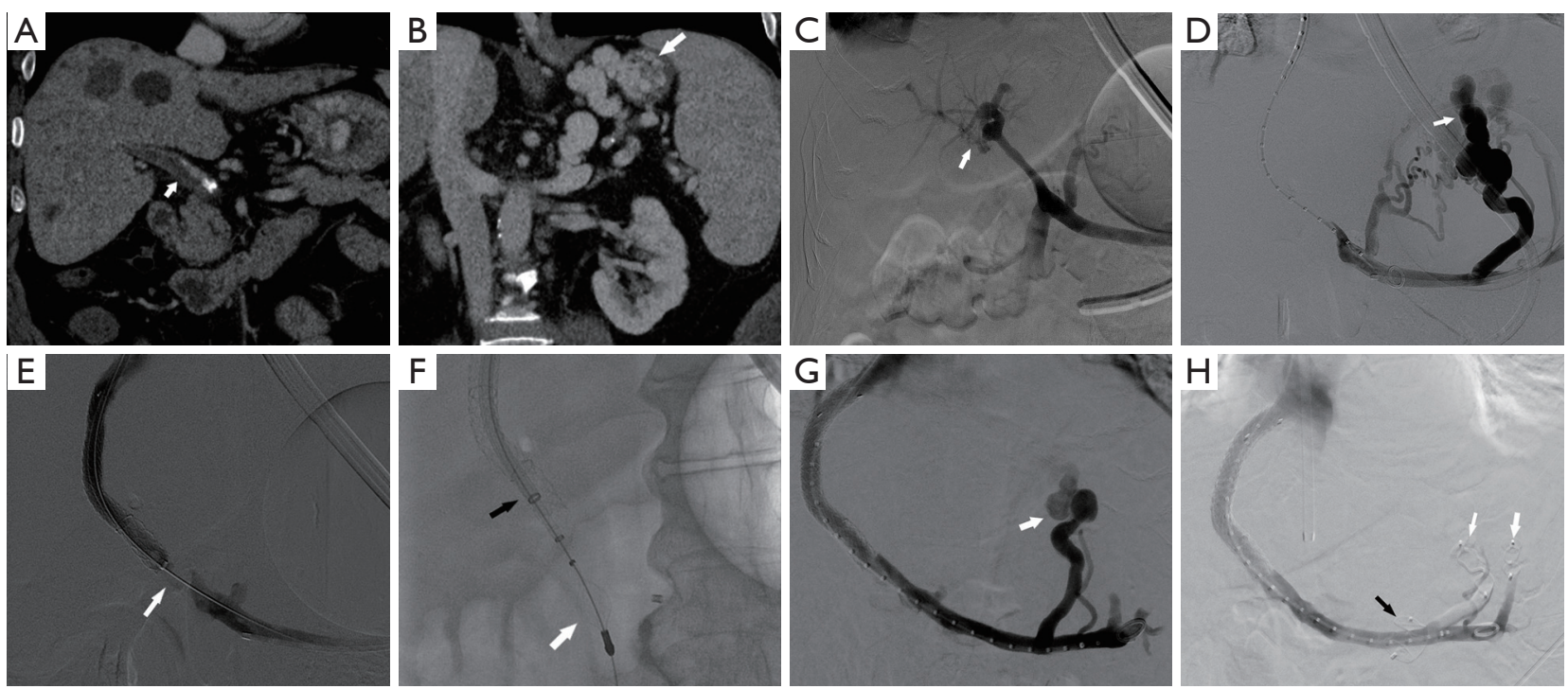

Figure 2 Case 2: M, 69, with ETOH cirrhosis and bleeding gastric varices requiring Sengstaken Blakemore tube in the stomach and esophagus. (A and B) are coronal CT scans of upper abdomen revealing partially occlusive thrombus in the portal vein and large gastric varices (marked by arrows). (C) is a splenoportal venogram obtained by catheter through the trans splenic route. It reveals partially occlusive thrombus in the main portal vein and poorly visualized intrahepatic portal venous branches, specifically in the right lobe (marked by arrow) due to chronic partial occlusion. (D) reveals presence of large gastric varix (marked by arrow) with left and posterior gastric afferent veins. (E) is a portal venogram after balloon venoplasty and mechanical thrombectomy with Angiojet thrombectomy device and TIPS placement. It reveals presence of thrombus in the main portal vein (marked by arrow) proximal to the TIPS. (F) shows Flow Triever2 catheter and disk (marked by white arrow) and a 20 French Flow Triever 20 aspiration catheter (marked by black arrow). (G) is splenoportal venogram status post mechanical thrombectomy, revealing complete resolution of the thrombus in the main portal vein and persistence of gastric varices after removal of the Sengstaken Blakemore tube. $(\mathrm{H})$ is splenoportal venogram obtained after PARTO procedure with occlusion of the descending inferior phrenic vein with amplatz vascular plug (marked by black arrow) and embolization of the afferent posterior gastric veins with amplatz vascular plugs (marked by white arrows) after embolization of the varix with gelfoam slurry. Venogram reveals complete non visualization of the gastric varices and patent main portal vein. TIPS, transjugular intrahepatic portosystemic shunt; ETOH, ethanol; PARTO, plug-assisted retrograde transvenous obliteration.

17 patients, with no patient developing portal hypertensive complications during follow-up (43). Significant shortand long-term benefits with thrombolysis were noted in a meta-analysis performed by Cheng et al. (47). The authors demonstrated favorable clinical and radiological outcomes, with a symptom improvement rate of $86 \%$ and a partial/ complete recanalization rate of $84 \%$. Frequently, there is no correlation between clinical and radiological improvement, as noted in a study by Hollingshead et al. (48), in which symptomatic improvement was noted in $93.7 \%$ of patients in spite of radiological improvement in just $68.8 \%$.

\section{TIPS placement}

TIPS placement is frequently used in patients with PVT and cirrhosis after mechanical thrombectomy and/ or chemical thrombolysis. While thrombectomy and thrombolysis resolve the acute thrombus, these techniques does not address the underlying issue of increased intrahepatic vascular resistance and slow blood flow in the main portal vein. Therefore, placement of a TIPS allows for reduction of the pressure in the main portal vein and provides outflow for the blood without stagnation (49).

In a large retrospective study evaluating the safety and efficacy of TIPS placement in patients with PVT, Luca et al. (49) reported $100 \%$ technical success of TIPS insertion without any procedure-related mortalities. They also reported impressive rates of recanalization of the portal vein after TIPS placement. Of the 70 patients with acute PVT who underwent TIPS placement, complete recanalization of the portal vein was achieved in 40 patients $(57 \%)$, partial recanalization with marked decrease in the PVT 
was observed in 21 patients (30\%), and no improvement was noted in 9 patients (13\%). Portal vein patency was maintained in 38 patients (95\%) at a mean follow-up of 20.7 months, with rethrombosis of the portal vein noted in 2 patients (5\%) at 6 months and 1 patient at 26 months. These high recanalization rates and maintenance of patency at follow-up were observed in spite of a hypercoagulable state secondary to high incidence of congenital thrombofilic factors in $45 \%$ of the 38 patients. These findings reaffirm the role of TIPS placement for maintaining patency of the portal vein by improving flow and providing outflow, bypassing high intrahepatic portal pressures. This theory is also supported by a prospective cohort study demonstrating low portal blood flow velocity as an independent predictor of PVT (2). Although the retrospective study demonstrated the safety and efficacy of TIPS placement in this patient population, it was limited by the absence of a control group. The study did not provide a comparison of the efficacy of anticoagulation versus TIPS placement for the initial management of acute PVT in cirrhotic patients. Additionally, the question of whether TIPS placement can or should be offered to patients with PVT without clinical manifestations remains unanswered.

In a meta-analysis comparing the efficacy of anticoagulation and TIPS placement for PVT, 7 studies (4 for anticoagulation and 3 for TIPS placement) were included. Anticoagulation and TIPS placement were evaluated in 179 and 148 patients, respectively (50). The analysis demonstrated that both treatments led to improved recanalization rates when compared to a control group. However, the mortality benefit seen with anticoagulation was not seen with TIPS placement. Based on these findings, it appears that all patients with cirrhosis and PVT should be offered anticoagulation as a first-line therapy after variceal prophylaxis (in the absence of any contraindications). In patients who are not candidates for anticoagulation and in those with disease that is refractory to anticoagulation, TIPS placement provides an additional route for recanalization and is an effective second-line therapy $(51,52)$.

Placement of TIPS in patients with PVT is frequently challenging and should be performed by experienced physicians. Either percutaneous or endovascular ultrasound guidance or percutaneous guidewire placement in the portal vein as a target is helpful in guiding access to the portal vein from the hepatic vein. Once portal venous access has been obtained, mechanical thrombectomy and/or balloon venoplasty is recommended before the placement of TIPS. After the TIPS is placed and adequate flow through the portal vein and the TIPS has been established, a splenoportal venogram should be obtained to evaluate varices. If varices are present after TIPS placement and after an adequate portosystemic gradient has been achieved, embolization with coils/vascular plug should be considered (Figure 2).

\section{Chronic PVT with cavernous transformation}

If not resolved, acute PVT can evolve into chronic PVT, leading to cavernous transformation of the portal vein. This frequently leads to massive esophageal, gastric, gastroesophageal, or ectopic varices and potentially bleeding. Additionally, patients can have refractory ascites and/or hydrothorax. However, with adequate collateral circulation, many patients are completely asymptomatic. Complex portal vein recanalization through either transplenic or transhepatic access and placement of TIPS is typically used in these patients. Additionally, recanalization of the chronically occluded portal vein with TIPS placement in posttransplant patients can help to restore portal vasculature, enabling normal physiological anastomosis (52). Considering the complexity of this procedure, the TIPS placement should be performed by experienced IR physicians with good clinical and surgical backup facilities.

\section{Proposed algorithm for acute PVT in cirrhosis}

Management of PVT in cirrhosis is complex, as multiple invasive and noninvasive treatment options must be considered, along with multiple variables that can affect the treatment outcomes. The lack of randomized controlled trials also complicates management strategies. Here, we propose a preliminary algorithm to approach acute PVT in cirrhotic patients based on our experience and review of the literature. We recognize that this algorithm is limited by a lack of randomized trials for validation. Additionally, factors that may affect the treatment options include status of varices, transplant status, extent and duration of thrombus, presence and severity of symptoms, renal dysfunction, and contraindication to anticoagulation (Figure 3).

\section{Conclusions}

PVT in cirrhosis is an increasingly recognized entity. The management of this condition is complex, as many variables affect which treatments can be used. Anticoagulation appears to be the optimal first-line treatment in patients with acute PVT but without bleeding varices or mesenteric 


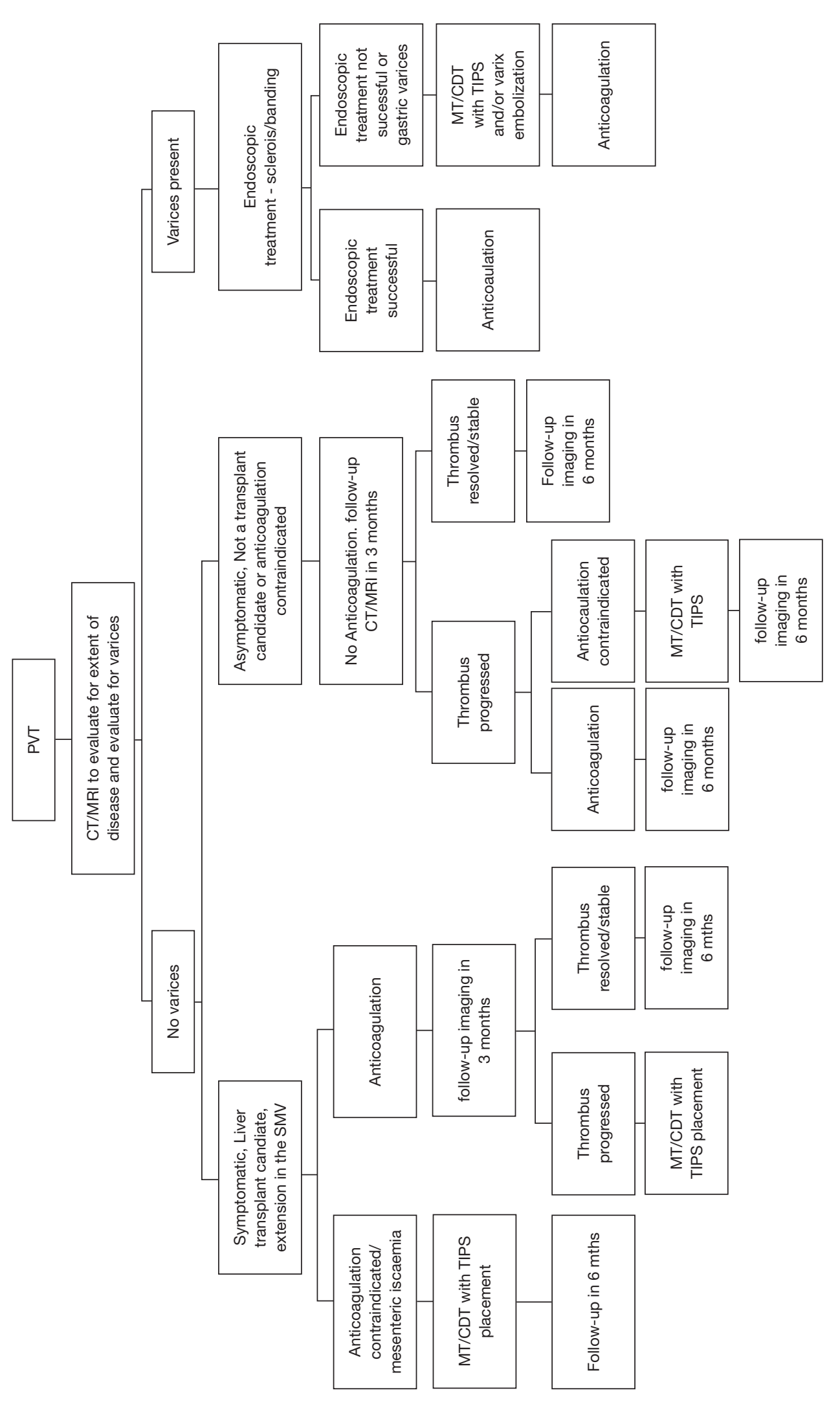

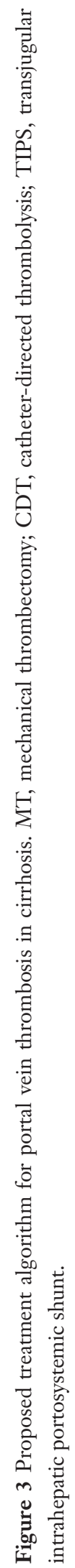


ischemia. Available minimally invasive treatments include various methods of mechanical thrombectomy, chemical thrombolysis, and TIPS placement with or without variceal embolization. No firm recommendations can be made because of the heterogeneity of available data and lack of randomized controlled trials. Multi-institutional randomized trials are urgently needed to evaluate the efficacy of various anticoagulants and their application in specific circumstances, as well as the timing and use of various minimally invasive therapies.

\section{Acknowledgments}

Funding: None.

\section{Footnote}

Reporting Checklist: The authors have completed the Narrative Review reporting checklist. Available at https:// cdt.amegroups.com/article/view/10.21037/cdt-21-98/rc

Conflicts of Interest: All authors have completed the ICMJE uniform disclosure form (available at https://cdt.amegroups. com/article/view/10.21037/cdt-21-98/coif). SP serves as an unpaid editorial board member of Cardiovascular Diagnosis and Therapy from September 2021 to August 2023. The other authors have no conflicts of interest to declare.

Ethical Statement: The authors are accountable for all aspects of the work in ensuring that questions related to the accuracy or integrity of any part of the work are appropriately investigated and resolved.

Open Access Statement: This is an Open Access article distributed in accordance with the Creative Commons Attribution-NonCommercial-NoDerivs 4.0 International License (CC BY-NC-ND 4.0), which permits the noncommercial replication and distribution of the article with the strict proviso that no changes or edits are made and the original work is properly cited (including links to both the formal publication through the relevant DOI and the license). See: https://creativecommons.org/licenses/by-nc-nd/4.0/.

\section{References}

1. Faccia M, Ainora ME, Ponziani FR, et al. Portal vein thrombosis in cirrhosis: Why a well-known complication is still matter of debate. World J Gastroenterol
2019;25:4437-51.

2. Zocco MA, Di Stasio E, De Cristofaro R, et al. Thrombotic risk factors in patients with liver cirrhosis: correlation with MELD scoring system and portal vein thrombosis development. J Hepatol 2009;51:682-9.

3. Francoz C, Valla D, Durand F. Portal vein thrombosis, cirrhosis, and liver transplantation. J Hepatol 2012;57:203-12.

4. Rodríguez-Castro KI, Porte RJ, Nadal E, et al. Management of nonneoplastic portal vein thrombosis in the setting of liver transplantation: a systematic review. Transplantation 2012;94:1145-53.

5. Noronha Ferreira C, Reis D, Cortez-Pinto H, et al. Anticoagulation in Cirrhosis and Portal Vein Thrombosis Is Safe and Improves Prognosis in Advanced Cirrhosis. Dig Dis Sci 2019;64:2671-83.

6. Loudin M, Ahn J. Portal Vein Thrombosis in Cirrhosis. J Clin Gastroenterol 2017;51:579-85.

7. Qi X, Han G, Fan D. Management of portal vein thrombosis in liver cirrhosis. Nat Rev Gastroenterol Hepatol 2014;11:435-46.

8. Valeriani E, Riva N, Di Nisio M, et al. Splanchnic Vein Thrombosis: Current Perspectives. Vasc Health Risk Manag 2019;15:449-61.

9. Thatipelli MR, McBane RD, Hodge DO, et al. Survival and recurrence in patients with splanchnic vein thromboses. Clin Gastroenterol Hepatol 2010;8:200-5.

10. Johnson GE, Allison SK, Shin DS. Acute portomesenteric venous thrombosis: Diagnosis and treatment. Dig Dis Interv 2018;2:249-55.

11. Alzubaidi S, Patel I, Saini A, et al. Current concepts in portal vein thrombosis: etiology, clinical presentation and management. Abdom Radiol (NY) 2019;44:3453-62.

12. Turkeltaub JA, Kriss M. Management of Portal and Mesenteric Venous Thrombosis. Dig Dis Interv 2020;4:122-33.

13. Yerdel MA, Gunson B, Mirza D, et al. Portal vein thrombosis in adults undergoing liver transplantation: risk factors, screening, management, and outcome. Transplantation 2000;69:1873-81.

14. Sarin SK, Philips CA, Kamath PS, et al. Toward a Comprehensive New Classification of Portal Vein Thrombosis in Patients With Cirrhosis. Gastroenterology 2016;151:574-577.e3.

15. Mikuła T, Kozłowska J, Stańczak W, et al. Serum ADAMTS-13 Levels as an Indicator of Portal Vein Thrombosis. Gastroenterol Res Pract 2018;2018:3287491. 16. Violi F, Ferro D, Basili S, et al. Ongoing prothrombotic 
state in the portal circulation of cirrhotic patients. Thromb Haemost 1997;77:44-7.

17. Wosiewicz P, Żorniak M, Hartleb M, et al. Portal vein thrombosis in cirrhosis is not associated with intestinal barrier disruption or increased platelet aggregability. Clin Res Hepatol Gastroenterol 2016;40:722-9.

18. DeLeve LD, Valla DC, Garcia-Tsao G, et al. Vascular disorders of the liver. Hepatology 2009;49:1729-64.

19. Stine JG, Shah NL, Argo CK, et al. Increased risk of portal vein thrombosis in patients with cirrhosis due to nonalcoholic steatohepatitis. Liver Transpl 2015;21:1016-21.

20. Zhang D, Hao J, Yang N. Protein C and D-dimer are related to portal vein thrombosis in patients with liver cirrhosis. J Gastroenterol Hepatol 2010;25:116-21.

21. Cheng XQ, Zuo CJ, Tian JM, et al. Portal vein aneurysms with multiple associated findings. Vasa 2010;39:312-8.

22. Thornburg B. Acute Portal Vein Thrombosis. Dig Dis Interv 2017;1:248-53.

23. Riva N, Ageno W. Clinical manifestations and imaging tools in the diagnosis of splanchnic and cerebral vein thromboses. Thromb Res 2018;163:252-9.

24. Loffredo L, Pastori D, Farcomeni A, et al. Effects of Anticoagulants in Patients With Cirrhosis and Portal Vein Thrombosis: A Systematic Review and Meta-analysis. Gastroenterology 2017;153:480-487.e1.

25. Cerini F, Gonzalez JM, Torres F, et al. Impact of anticoagulation on upper-gastrointestinal bleeding in cirrhosis. A retrospective multicenter study. Hepatology 2015;62:575-83.

26. Qi X, De Stefano V, Li H, et al. Anticoagulation for the treatment of portal vein thrombosis in liver cirrhosis: a systematic review and meta-analysis of observational studies. Eur J Intern Med 2015;26:23-9.

27. Pettinari I, Vukotic R, Stefanescu H, et al. Clinical Impact and Safety of Anticoagulants for Portal Vein Thrombosis in Cirrhosis. Am J Gastroenterol 2019;114:258-66.

28. Under the auspices of the Italian Association for the Study of Liver Diseases (AISF) and the Italian Society of Internal Medicine (SIMI). Hemostatic balance in patients with liver cirrhosis: Report of a consensus conference. Dig Liver Dis 2016;48:455-67.

29. La Mura V, Braham S, Tosetti G, et al. Harmful and Beneficial Effects of Anticoagulants in Patients With Cirrhosis and Portal Vein Thrombosis. Clin Gastroenterol Hepatol 2018;16:1146-1152.e4.

30. Delgado MG, Seijo S, Yepes I, et al. Efficacy and safety of anticoagulation on patients with cirrhosis and portal vein thrombosis. Clin Gastroenterol Hepatol 2012;10:776-83.

31. Amitrano L, Guardascione MA, Menchise A, et al. Safety and efficacy of anticoagulation therapy with low molecular weight heparin for portal vein thrombosis in patients with liver cirrhosis. J Clin Gastroenterol 2010;44:448-51.

32. Francoz C, Belghiti J, Vilgrain V, et al. Splanchnic vein thrombosis in candidates for liver transplantation: usefulness of screening and anticoagulation. Gut 2005;54:691-7.

33. Turon F, Hernández-Gea V, García-Pagán JC. Portal vein thrombosis: yes or no on anticoagulation therapy. Curr Opin Organ Transplant 2018;23:250-6.

34. Raja K, Jacob M, Asthana S. Portal vein thrombosis in cirrhosis. J Clin Exp Hepatol 2014;4:320-31.

35. Kwon J, Koh Y, Yu SJ, et al. Low-molecular-weight heparin treatment for portal vein thrombosis in liver cirrhosis: Efficacy and the risk of hemorrhagic complications. Thromb Res 2018;163:71-6.

36. Weinberg EM, Palecki J, Reddy KR. Direct-Acting Oral Anticoagulants (DOACs) in Cirrhosis and CirrhosisAssociated Portal Vein Thrombosis. Semin Liver Dis 2019;39:195-208.

37. Chamarthy MR, Anderson ME, Pillai AK, et al. Thrombolysis and Transjugular Intrahepatic Portosystemic Shunt Creation for Acute and Subacute Portal Vein Thrombosis. Tech Vasc Interv Radiol 2016;19:42-51.

38. Jun KW, Kim MH, Park KM, et al. Mechanical thrombectomy-assisted thrombolysis for acute symptomatic portal and superior mesenteric venous thrombosis. Ann Surg Treat Res 2014;86:334-41.

39. Uflacker R. Applications of percutaneous mechanical thrombectomy in transjugular intrahepatic portosystemic shunt and portal vein thrombosis. Tech Vasc Interv Radiol 2003;6:59-69.

40. Lichtenberg MKW, Stahlhoff S, Młyńczak K, et al. Endovascular mechanical thrombectomy versus thrombolysis in patients with iliofemoral deep vein thrombosis - a systematic review and meta-analysis. Vasa 2021;50:59-67.

41. Cai G, Li C, Hua Z, et al. AngioJet Aspiration Thrombectomy Combined with Transcatheter Thrombolysis in Treatment of Acute Portal Venous Systemic Thrombosis. Ann Vasc Surg 2020;66:362-9.

42. Seedial SM, Mouli SK, Desai KR. Acute Portal Vein Thrombosis: Current Trends in Medical and Endovascular Management. Semin Intervent Radiol 2018;35:198-202.

43. Partovi S, Kalva SP, Walker TG, et al. Long term followup of endo-vascular recanalization of chronic inferior vena 
cava occlusion secondary to inferior vena cava filters. Vasa 2017;46:121-6.

44. Klinger C, Riecken B, Schmidt A, et al. Transjugular local thrombolysis with/without TIPS in patients with acute non-cirrhotic, non-malignant portal vein thrombosis. Dig Liver Dis 2017;49:1345-52.

45. Skeik N, Gits CC, Ehrenwald E, et al. Fibrinogen level as a surrogate for the outcome of thrombolytic therapy using tissue plasminogen activator for acute lower extremity intravascular thrombosis. Vasc Endovascular Surg 2013;47:519-23.

46. Abdel-Aal AK, Ezzeldin IB, Hamed MF, et al. Endovascular treatment of acute portal vein thrombosis using ultrasound-accelerated catheter-directed thrombolysis. Vasc Endovascular Surg 2014;48:460-5.

47. Cheng Q, Tree K. Systematic Review of Thrombolysis Therapy in the Management of Non-CirrhosisRelated Portal Vein Thrombosis. J Gastrointest Surg 2021;25:1579-90.

48. Hollingshead M, Burke CT, Mauro MA, et al.

Transcatheter thrombolytic therapy for acute mesenteric

Cite this article as: Gadani S, Partovi S, Levitin A, Zerona N, Sengupta S, D’Amico G, Diago Uso T, Menon KVN, Quintini C. Narrative review of portal vein thrombosis in cirrhosis: pathophysiology, diagnosis, and management from an interventional radiology perspective. Cardiovasc Diagn Ther 2022;12(1):135-146. doi: 10.21037/cdt-21-98 and portal vein thrombosis. J Vasc Interv Radiol 2005;16:651-61.

49. Luca A, Miraglia R, Caruso S, et al. Short- and long-term effects of the transjugular intrahepatic portosystemic shunt on portal vein thrombosis in patients with cirrhosis. Gut 2011;60:846-52.

50. Davis JPE, Ogurick AG, Rothermel CE, et al. Anticoagulation and Transjugular Intrahepatic Portosystemic Shunting for Treatment of Portal Vein Thrombosis in Cirrhosis: A Systematic Review and Meta-Analysis. Clin Appl Thromb Hemost 2019;25:1076029619888026.

51. Stine JG, Wang J, Shah PM, et al. Decreased portal vein velocity is predictive of the development of portal vein thrombosis: A matched case-control study. Liver Int 2018;38:94-101.

52. Thornburg B, Desai K, Hickey R, et al. Pretransplantation Portal Vein Recanalization and Transjugular Intrahepatic Portosystemic Shunt Creation for Chronic Portal Vein Thrombosis: Final Analysis of a 61-Patient Cohort. J Vasc Interv Radiol 2017;28:1714-1721.e2. 